\title{
Tear film inflammatory mediators during continuous wear of contact lenses and corneal refractive therapy
}

\author{
Javier González-Pérez, ${ }^{1,2}$ César Villa-Collar, ${ }^{3}$ Tomás Sobrino Moreiras, ${ }^{4}$ \\ Isabel Lema Gesto, ${ }^{4,5}$ José Manuel González-Méijome, ${ }^{1,6}$ \\ María Teresa Rodríguez-Ares, ${ }^{5}$ Manuel Parafita, ${ }^{1,5}$
}

\begin{abstract}
${ }^{1}$ Ocular Surface and Contact Lens Research Laboratory. Faculty of Optometry, University of Santiago de Compostela, Coruña, Spain

${ }^{2}$ Department of Applied Physics (Optometry), University of Santiago de Compostela, Santiago de Compostela, Spain ${ }^{3}$ Department of Optometry, Ophthalmology Clinic

Novovision, Madrid, Spain ${ }^{4}$ Clinic Neuroscience Research Laboratory, CHUS, Santiago de Compostela, Spain

${ }^{5}$ Department of Surgery (Ophthalmology), University of Santiago de Compostela,

Santiago de Compostela, Spain ${ }^{6}$ Clinical and Experimental Optometry Research Laboratory, Center of Physics (Optometry), School of Sciences, University of Minho, Braga, Portugal
\end{abstract}

\section{Correspondence to}

Dr Javier González-Pérez, Ocular Surface and Contact Lens Research Laboratory, Faculty of Optics and Optometry, University of Santiago de Compostela, 15782 A Coruña, Spain;

javier.gonzalez@usc.es

Accepted 12 May 2012
ABSTRACT

Objectives To study changes in tear film inflammatory mediators following continuous wear of silicone-hydrogel lenses and corneal refractive therapy with reverse geometry contact lenses.

Design A prospective, case-control study.

Methods Twenty-eight subjects had worn siliconehydrogel lenses on a 30-night continuous wear basis. Thirty-two subjects had worn corneal refractive therapy lenses on an overnight basis. Thirty-two matched control subjects were also recruited. Tear samples were obtained 12 months after initial fitting and assayed using ELISA for cytokines (interleukin (IL)-6 and IL-8), matrix metalloproteinase 9 (MMP-9) and epidermal growth factor (EGF).

Results EGF was significantly increased 12 months after both interventions. IL-6, IL-8 and MMP-9 were significantly increased only after corneal refractive therapy. The inflammatory response for the corneal refractive therapy patients was found to be associated with the degree of myopia corrected and the presence of corneal staining. Moreover, an increased level of MMP-9 and EGF was found to be associated with the presence of corneal-pigmented arc in the corneal refractive therapy group.

Conclusions This research showed long-term increased tear levels of inflammatory markers in subjects wearing corneal refractive therapy lenses when compared with continuous wear of silicone-hydrogel lenses or no lens wear.

Continuous wear of contact lenses offers a flexible and reversible method to correct myopia. The short and long-term effects of this wear of soft lenses on the eye have been well documented. ${ }^{1}$ Complications induced by the continuous wear of contact lenses have been described by many investigators and were attributed, among other things, to three main potential aetiologies: hypoxia, mechanical effect and cytokine-mediated effects. ${ }^{2} 3$ As a result of the high oxygen transmissibility, lens movement and wetting performance of new continuous wear silicone-hydrogel lenses, a wearing schedule of up to 30 nights is now feasible. ${ }^{4}$ Numerous reports have recently been published on the clinical and subjective findings with silicone-hydrogel lenses, but few have specifically studied the impact on the level of tear inflammatory mediators following continuous wear. ${ }^{13}$

Corneal refractive therapy, also known as overnight orthokeratology, is an alternative use of reverse geometry contact lenses, intended to induce temporary flattening in the central corneal curvature producing short-term reduction in myopia. ${ }^{5}$ The introduction of the reverse geometry lens and modern rigid gas-permeable (RGP) lens materials has led to safer and more predictable overnight corneal refractive therapy. A number of studies has shown the efficacy of overnight corneal refractive therapy in reducing myopic refractive error and thus providing improved unaided visual acuity. ${ }^{6-8}$ Moreover, recent studies have shown the potential of this method to slow down myopia progression. ${ }^{9}$ The clinical efficacy of this technique for the reduction of low myopia is now well established, but little is known about the underlying corneal events associated with the significant central corneal flattening induced. Inflammation and infection are two major concerns for contact lens practitioners. Although several cases of microbial keratitis have been reported, there are no consistent data regarding its risk for corneal refractive therapy patients. ${ }^{10}$ Moreover, the fact that corneal refractive therapy lenses retain more bacteria than alignment fit RGP lenses ${ }^{11}$ may increase the risk of infection and inflammation. The evaluation of levels of inflammatory mediators at the ocular surface is thus important to know if the cornea undergoing corneal refractive therapy could be compromised.

Mechanical trauma to the cornea has been related to the upregulation of inflammatory mediators such as interkeukin (IL)-8 and epidermal growth factor (EGF) during extended wear of highDk silicone-hydrogel or RGP contact lenses. ${ }^{3}$ Daily wear of RGP contact lenses also induces the overexpression of proinflammatory molecules such as IL-6, tumour necrosis factor alpha (TNF $\alpha$ ) and IL-10. ${ }^{12}$ Hypoxic stress during overnight wear has also proved to increase the release of proinflammatory mediators such as IL-8. ${ }^{13}$ The mechanical forces acting during corneal refractive therapy ${ }^{14}$ along with the hypoxic stimuli ${ }^{15}$ and the reduced tear turnover behind the lens ${ }^{16}$ could upregulate the release of inflammatory mediators. As high-Dk materials exceed the Holden and Mertz criterion for oxygen transmissibility required to overcome the effects of hypoxia during overnight wear, this aspect could be assessed by comparison between both silicone-hydrogel and corneal refractive therapy overnight wearing lenses. ${ }^{17}$

The aim of the present study was to measure the concentration of several tear markers involved in the corneal wound healing response to evaluate whether a specific molecular marker follows 
significant changes during corneal refractive therapy and continuous wear of contact lenses in the long-term period. Proinflammatory cytokine IL-6, chemokine IL-8, EGF and matrix metalloproteinase 9 (MMP-9) production were measured in tears of myopic patients 12 months after both treatments and compared with a control group.

\section{MATERIALS AND METHODS}

\section{Subjects and contact lenses}

The present study was conducted at the Ocular Surface and Contact Lens Research Laboratory, University of Santiago de Compostela (Galicia, Spain) and Ophthalmology Clinic Novovision (Madrid, Spain). The study comprised 60 right eyes from 60 healthy subjects aged $26.4 \pm 6.1$ years. Thirty-two subjects (16 women and 16 men) aged 27.0 \pm 7.4 years had worn Paragon CRT (Paragon Vision Sciences, Inc, Mesa, Arizona, USA) for 12 months. Twenty-eight subjects (16 women and 12 men) aged $25.5 \pm 5.0$ years had worn Lotrafilcon A silicone-hydrogel lenses (Ciba Vision, Duluth, Georgia, USA) on a continuous wear basis (30 nights) for 12 months. All subjects were asymptomatic and before contact lens fitting were examined for any inflammatory disease. Thirty-two subjects (16 women and 16 men) aged $26.7 \pm 5.1$ years, who had never worn contact lenses, were also recruited as a control group. The exclusion criteria were the presence or history of any systemic or ocular disorder, including ocular surgery, trauma or disease.

After tear collection, a full ophthalmic examination was conducted for all subjects in every group. This included routine evaluation of visual acuity, refractive error and slit-lamp biomicroscopy with particular attention to the occurrence of adverse events or mild changes such as pigmented ring or corneal staining.

\section{Inflammatory mediator assays}

Tear samples were collected using $50 \mu \mathrm{l}$ of calibrated glass microcapillaries (Micro Titertube natural 845-TP; TTE Laboratories, Hopkinton, Massachusetts, USA), from the inferior temporal tear meniscus by capillary flow. From each subject, at least $15 \mu \mathrm{l}$ of tears were collected. Care was taken to avoid touching the lid margins or the corneal surface. While collecting tears, the flow rate was controlled, and only samples with a flow rate of $1-10 \mu \mathrm{l} / \mathrm{min}$ were used in this investigation to avoid the dilution effect caused by a possible hypersecretion of tears during the process. Microcapillary tubes were immediately transferred to Falcon tubes and stored at $-80^{\circ} \mathrm{C}$ until they were assayed.
Tears were examined for IL-6, IL-8, MMP-9 and EGF. All of these molecules have been implicated in the corneal wound healing response and represent constant components of the tear fluid.

The concentration of inflammatory mediators was measured using commercially available quantitative sandwich ELISA kits (Quantikine; R\&D Systems Europe, Abingdon, UK). The samples were centrifuged at $13000 \mathrm{rpm}$ for $5 \mathrm{~min}$ and supernatant transferred to new Falcon tubes. Sample preparation and analysis were performed according to the protocols supplied by the manufacturers. Samples were diluted 1:20, 1:80 and 1:100 for cytokines, EGF and MMP-9, respectively, with sample calibrator diluents (reagents provided with each ELISA kit), and final results were corrected according to the dilution factor. All determinations were carried out without knowledge of the sample characterisation by the technician (blind test).

\section{Statistical analysis}

The Kolmogorov-Smirnov test was applied to assess the normality of data distribution. Independent Mann-Whitney $U$ tests were used to compare differences in cytokines, MMP-9 and EGF concentrations in subjects wearing silicone-hydrogel or Paragon CRT contact lenses and controls. Kruskal-Wallis analysis was used to evaluate potential differences with the amount of refractive error in subjects wearing contact lenses compared with control data. Data were analysed using the statistical package PASW version 18.0. The level of $p<0.05$ was accepted as statistically significant.

\section{RESULTS \\ Subjects' demographics}

Table 1 shows subjects' demographics and the refractive status of the right eyes at the start of the study. Both contact lens groups were matched in terms of baseline age, refractive error and treatment duration. No statistically significant differences were found between groups for the initial refractive error in terms of sphere, astigmatism, mean spherical equivalent or keratometric radius $(p>0.05)$. No adverse events were observed with the exception of some minor changes such as trace to moderate corneal staining in both contact lens wear groups and pigmented ring in the corneal refractive therapy group. These findings will be used to correlate their presence with the upregulation of certain molecules under evaluation.

\section{Inflammatory mediators in control subjects and contact lens wearers}

Levels of inflammatory mediators and significance after corneal refractive therapy and continuous wear compared with controls

Table 1 Baseline descriptive statistics for normal subjects and contact lens wearers at the start of the study

\begin{tabular}{lcccc}
\hline & Control (mean \pm SD) & Si-H (mean \pm SD) & CRT (mean \pm SD) & p Value \\
\hline Age (years) & $26.72 \pm 5.12$ & $25.46 \pm 5.04$ & $27.00 \pm 7.38$ & $0.275^{*}$ \\
Initial sphere (D) & $-2.57 \pm 1.34$ & $-2.43 \pm 1.37$ & $-2.58 \pm 1.04$ & $0.194 \dagger$ \\
Initial cylinder (D) & $-0.50 \pm 0.42$ & $-0.54 \pm 0.43$ & $-0.25 \pm 0.33$ & $0.054 \dagger$ \\
Spherical equivalent (D) & $-2.76 \pm 1.28$ & $-2.77 \pm 1.54$ & $-2.69 \pm 1.07$ & $0.498 \dagger$ \\
CC centre (D) & $43.87 \pm 1.57$ & $44.28 \pm 0.98$ & $43.89 \pm 1.84$ & $0.545^{*}$ \\
CC cord 2 mm (D) & $43.54 \pm 1.70$ & $43.92 \pm 0.98$ & $43.64 \pm 1.72$ & $0.651^{*}$ \\
CC cord 4 mm (D) & $42.93 \pm 1.56$ & $43.33 \pm 0.95$ & $43.04 \pm 1.77$ & $0.623^{*}$ \\
CC cord 6 mm (D) & $41.63 \pm 1.31$ & $42.42 \pm 0.93$ & $41.64 \pm 1.73$ & $0.230^{*}$ \\
Time of study (months) & - & $11.29 \pm 1.49$ & $12.03 \pm 2.06$ & $0.152 \dagger$ \\
$\mathrm{n}$ (Sample) & 32 & 28 & 32 & $0.082 \dagger$ \\
\hline
\end{tabular}

*Analysis of variance (Bonferroni post-hoc).

†Kruskal-Wallis test.

CC, corneal curvature; CRT, corneal refractive therapy group; D, diopters; Si-H, silicone-hydrogel group. 
Table 2 Descriptive analysis and significance for tear marker levels in the samples collected from contact lens wearers compared with control values

\begin{tabular}{|c|c|c|c|c|c|}
\hline \multirow[b]{2}{*}{ Case-control } & \multirow{2}{*}{$\begin{array}{l}\text { Control }(n=32) \\
\text { Median } \pm \text { SD }\end{array}$} & \multicolumn{2}{|l|}{$\mathrm{Si}-\mathrm{H}(\mathrm{n}=28)$} & \multicolumn{2}{|l|}{ CRT $(n=32)$} \\
\hline & & Median \pm SD & p Value ${ }^{*}$ & Median \pm SD & p Value* \\
\hline IL-6 (pg/ml) & $2.2 \pm 0.5$ & $2.6 \pm 0.6$ & 0.076 & $4.7 \pm 1.2$ & $<0.001$ \\
\hline IL-8 (pg/ml) & $601.5 \pm 72.7$ & $659.5 \pm 105.1$ & 0.074 & $935.3 \pm 254.4$ & $<0.001$ \\
\hline EGF (pg/ml) & $698.1 \pm 271.6$ & $1536.6 \pm 492.6$ & $<0.001$ & $2348.7 \pm 629.7$ & $<0.001$ \\
\hline
\end{tabular}

*Mann-Whitney test.

CRT, corneal refractive therapy group; EGF, epidermal growth factor; IL, interleukin; MMP-9, matrix metalloproteinase 9; Si-H, silicone-hydrogel group.

are shown in table 2. Median values of IL-6 $(\mathrm{p}=0.076)$, IL-8 $(\mathrm{p}=0.074)$ and MMP-9 $(\mathrm{p}=0.068)$ were similar in the tear samples collected from the silicone-hydrogel lens wearers and controls. However, Mann-Whitney tests confirmed that the EGF concentration was significantly greater in the tear samples collected from the silicone-hydrogel lens wearers $(p<0.001)$ and the Paragon CRT lens wearers $(p<0.001)$ compared with those obtained from the control group. In addition, IL-6, IL-8 and MMP-9 were significantly greater in the tear samples collected from the Paragon CRT lens group $(p<0.001)$ compared with those obtained from the control or silicone-hydrogel subjects. Tear marker concentrations are presented graphically in figure 1, showing the median and IOR for the three subject groups.

The MMP-9 $(p=0.042)$ and EGF $(p<0.001)$ concentrations for the silicone-hydrogel group and the IL-6 $(p<0.001)$, IL- 8 $(p<0.001)$, MMP-9 $(p<0.001)$ and EGF $(p<0.001)$ tear levels for the CRT subjects were significantly associated with the degree of myopia correction. Likewise, the increase of both proinflammatory markers $(p<0.05)$ and EGF $(p=0.001)$ in the corneal refractive therapy group and the increased levels of EGF $(p=0.044)$ in the silicone-hydrogel group were also found to be associated with the presence of low to moderate fluorescein corneal staining. Values measured after treatment with contact lenses compared with controls can be graphically observed in figures $2-5$. This inflammatory upregulation associated with contact lens wear is much more significant in corneal refractive therapy subjects when compared with the silicone-hydrogel lens wearers or the control groups.

Another interesting clinical finding of this study is the presence of deposition, presumably of iron, in the corneal epithelium of $60 \%$ of the patients who have been undergoing treatment with overnight corneal refractive therapy for 12 months. When the magnitude of the inflammatory response was analysed in relation to the presence of iron deposition, MMP-9 and EGF were increased in eyes presenting this finding $(p=0.001$ and $p=0.039$, respectively; figure 6).

\section{DISCUSSION}

This study has demonstrated increased levels of inflammatory markers, matrix proteinase and EGF in the tears of subjects wearing Paragon CRT lenses after a 12-month wearing period. The magnitude of this response was found to be associated with the degree of myopia to be corrected and the presence of fluorescein corneal staining suggesting a Paragon CRT lens compressive effect and mechanical trauma as the main causes of the inflammatory upregulation. ${ }^{18} 19$

Cytokines play a major role during inflammation and host defence primarily by regulating the diverse functions of
Figure 1 Tear film markers during continuous wear of silicone-hydrogel contact lenses and corneal refractive therapy compared with controls. A significant increase in the levels of inflammatory mediators $(p<0.001)$ associated with corneal refractive therapy is particularly noteworthy. CRT, corneal refractive therapy group; $D$, diopters; EGF, epidermal growth factor; IL, interleukin; MMP-9, matrix metalloproteinase 9; $\mathrm{Si}-\mathrm{H}$, siliconehydrogel group.
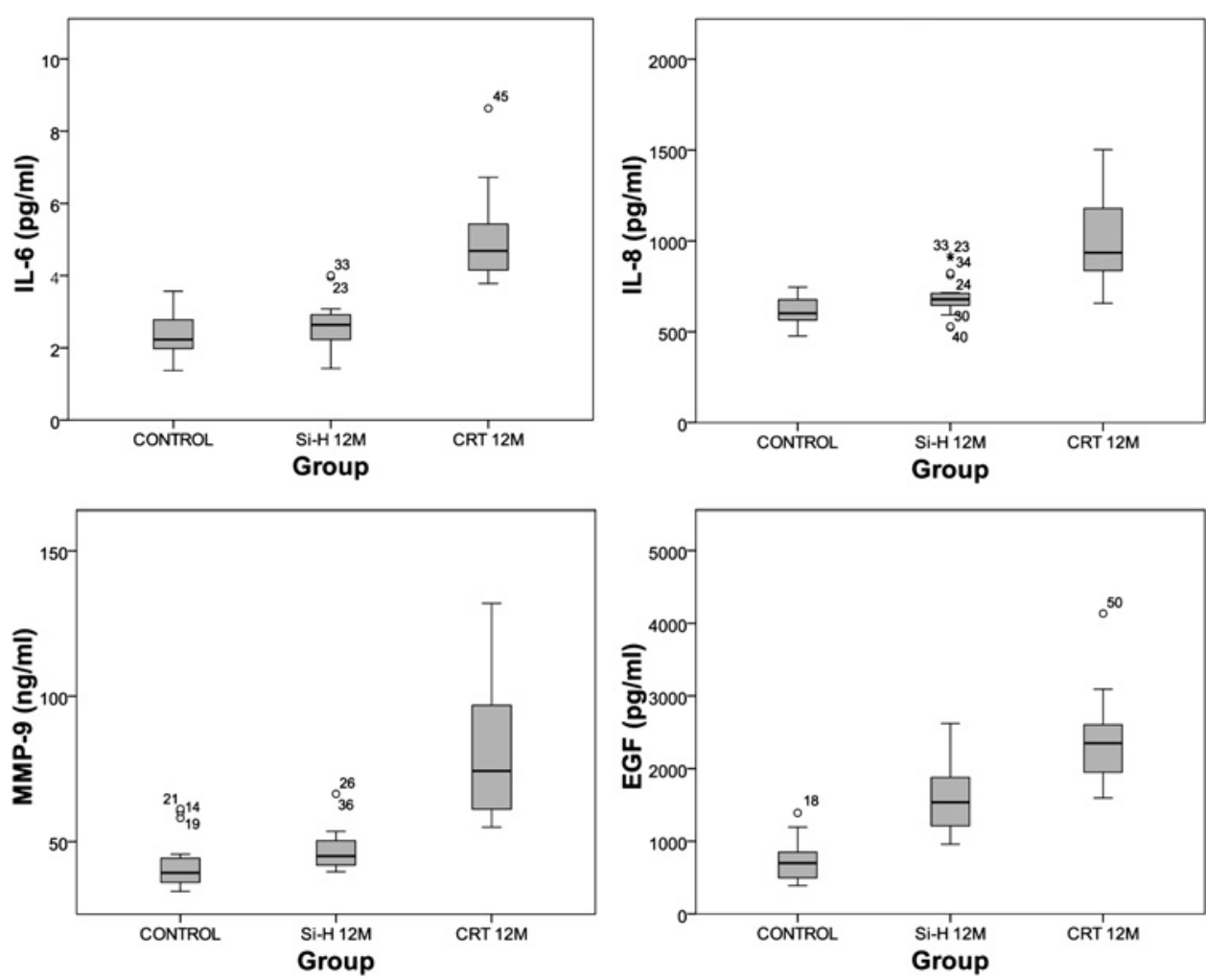
Figure 2 Box-plots representing tear film markers in samples collected from the continuous wear of siliconehydrogel contact lens group compared with controls. Only a significant increase in the levels of epidermal growth factor $(p<0.001)$ can be observed in connection with the amount of treated myopia for silicone-hydrogel lens wearers. D, diopters; EGF, epidermal growth factor; IL, interleukin; MMP-9, matrix metalloproteinase 9; $\mathrm{Si}-\mathrm{H}$, silicone-hydrogel group.
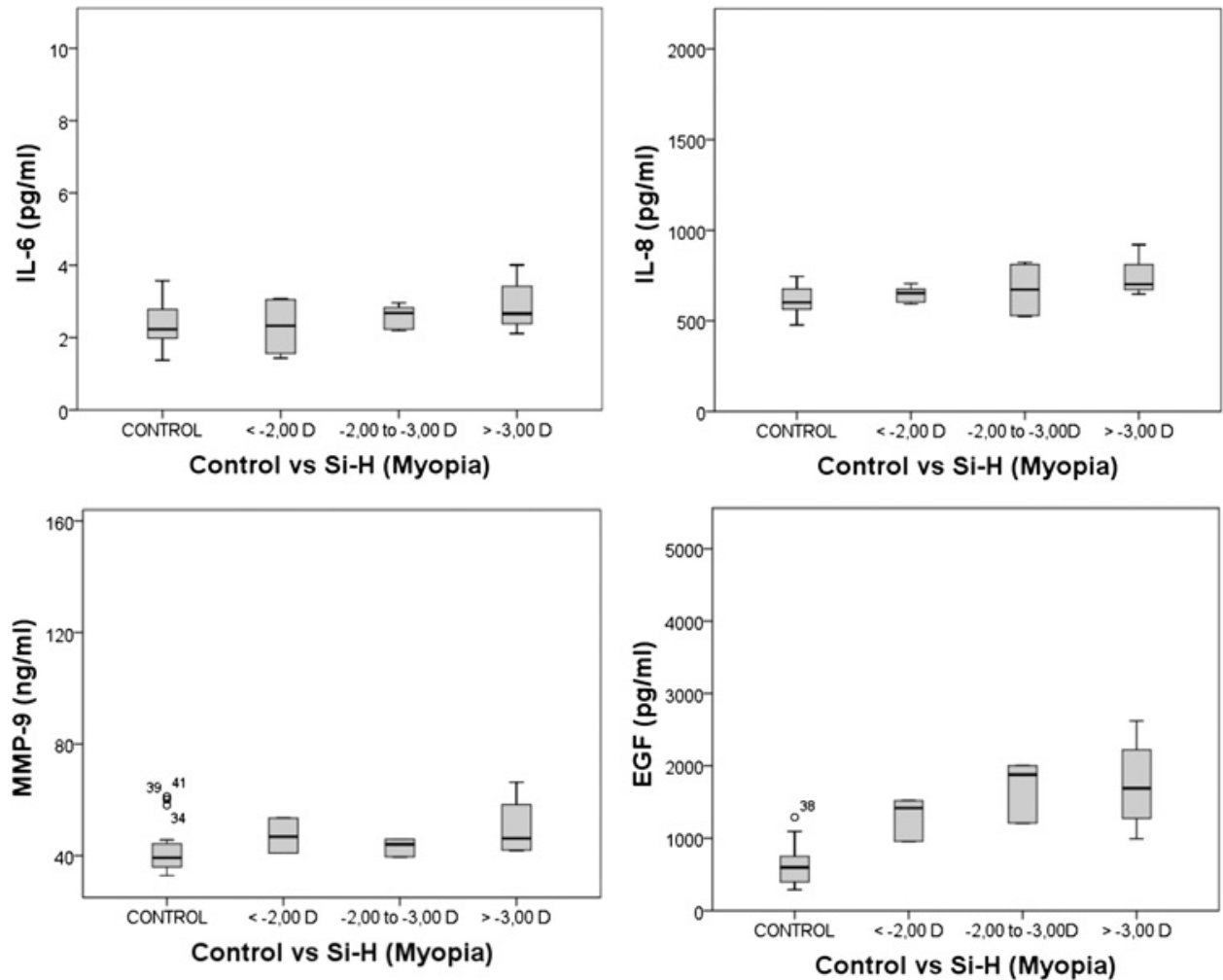

phagocytes. Cytokines also regulate the expression of several inflammatory mediators by local cells and are therefore important in the local regulation of inflammation. Contact lensinduced trauma to the epithelium could result in the increased release of inflammatory mediators, facilitating the loss/redistribution of corneal fibroblasts ${ }^{320}$ and cell proliferation. ${ }^{21}$ Previous reports of inflammatory mediators in tears of contact lens wearers have shown the altered presence of histamine, granulocyte macrophage colony-stimulating factor, TNF $\alpha$, IL-6, IL-8, leukotriene B4, MMP-9 and EGF. ${ }^{3} 12$ In this study, it was found that IL-6, IL-8, MMP-9 and EGF tear levels were greater in the samples collected from the CRT group compared with the
Figure 3 Box-plots representing tear film markers in samples collected from the corneal refractive therapy group compared with controls. A significant increase in the levels of all inflammatory mediators $(p<0.001)$ can be observed in connection with the amount of treated myopia for corneal refractive therapy subjects. CRT, corneal refractive therapy group; $\mathrm{D}$, diopters; EGF, epidermal growth factor; IL, interleukin; MMP-9, matrix metalloproteinase 9 .
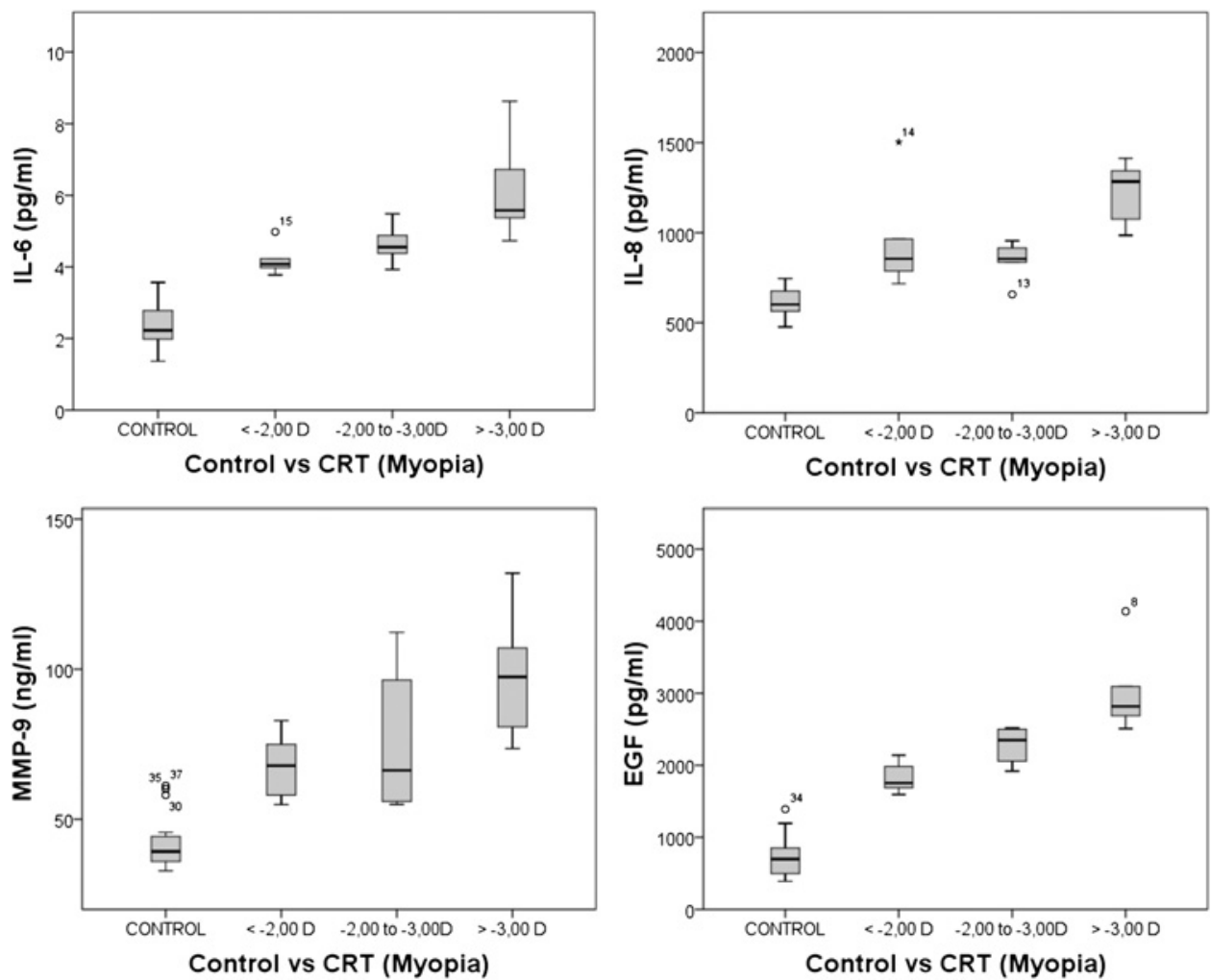
Figure 4 Box-plots representing tear film markers in samples collected from the continuous wear of siliconehydrogel contact lens group compared with controls. Only a significant increase in the levels of epidermal growth factor $(p<0.001)$ can be observed in connection with the presence of fluorescein corneal staining for silicone-hydrogel lens wearers. EGF, epidermal growth factor; IL, interleukin; MMP-9, matrix metalloproteinase 9; $\mathrm{Si}-\mathrm{H}$, silicone-hydrogel group.
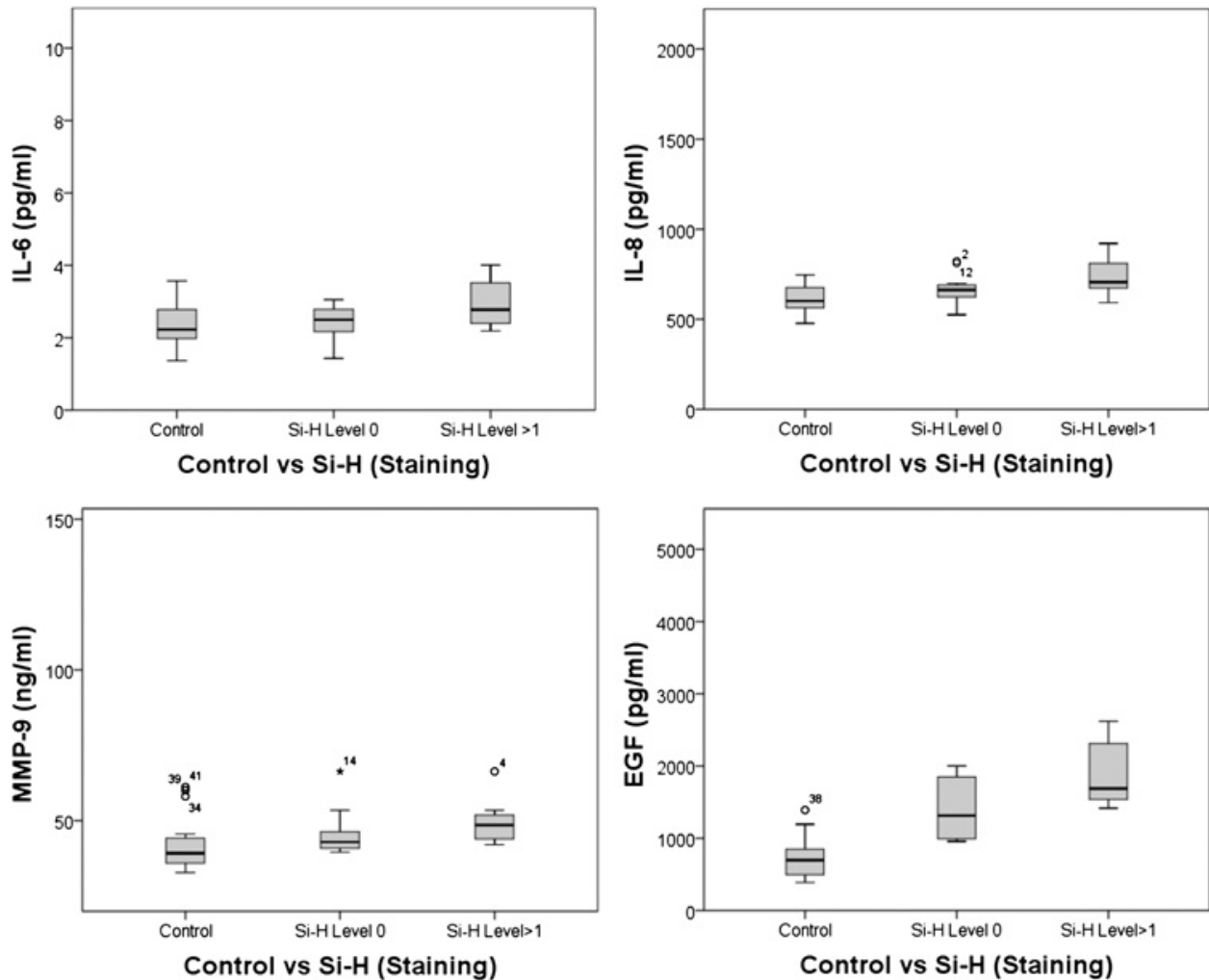

samples obtained from the control or silicone-hydrogel extended wearers.

IL-6 is a pleiotropic proinflammatory cytokine synthesised by various cells, such as fibroblasts, endothelial cells and keratinocytes, in response to proinflammatory stimuli including IL- 1 and TNF $\alpha$. IL- 6 stimulates epithelial migration in the cornea ${ }^{23}$ and can also induce the expression of matrix metalloproteinases. ${ }^{24}$ IL-6 is a constant component of the normal human tear fluid ${ }^{25}$ and has been found to be upregulated after corneal epithelial aggression including RGP lens wear. ${ }^{12} 26$ That work found a $\times 2.1$ increase of IL- 6 in tear samples of corneal refractive therapy subjects, which is in agreement with previous studies
Figure 5 Box-plots representing tear film markers in samples collected from the corneal refractive therapy group compared with controls. A significant increase in the levels of all inflammatory mediators $(p<0.001)$ can be observed in connection with the presence of fluorescein corneal staining for corneal refractive therapy subjects. CRT, corneal refractive therapy group; EGF, epidermal growth factor; IL, interleukin; MMP-9, matrix metalloproteinase 9.
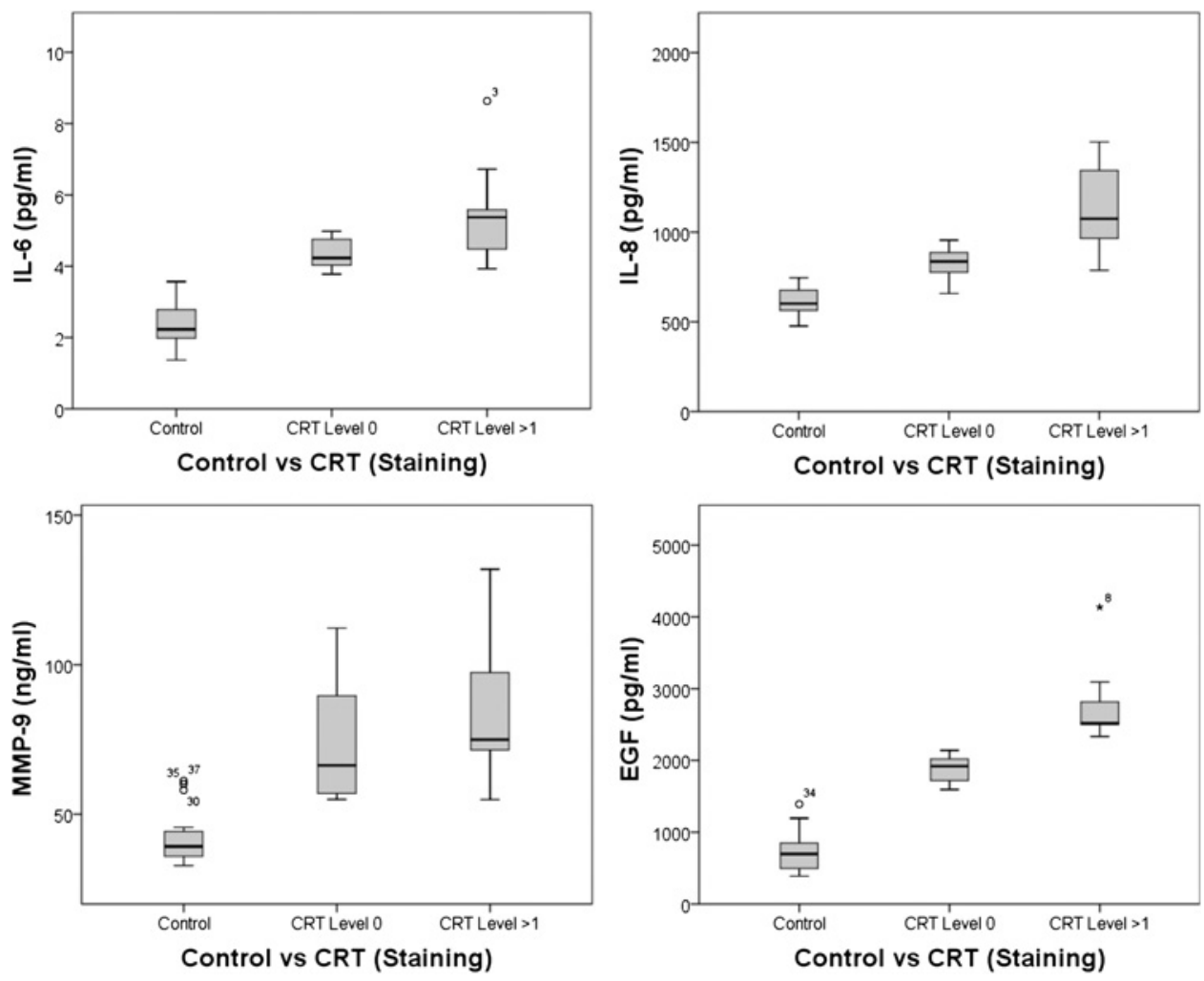
Figure 6 Box-plots representing tear film markers in samples collected from the corneal refractive therapy group compared with controls. A significant increase in the levels of matrix metalloproteinase $9(p=0.001)$ can be observed in connection with the presence of iron deposition in the corneal epithelium for corneal refractive therapy patients. CRT, corneal refractive therapy group; EGF, epidermal growth factor; IL, interleukin; MMP-9, matrix metalloproteinase 9; NPA, no pigmented arc; WPA, with pigmented arc.
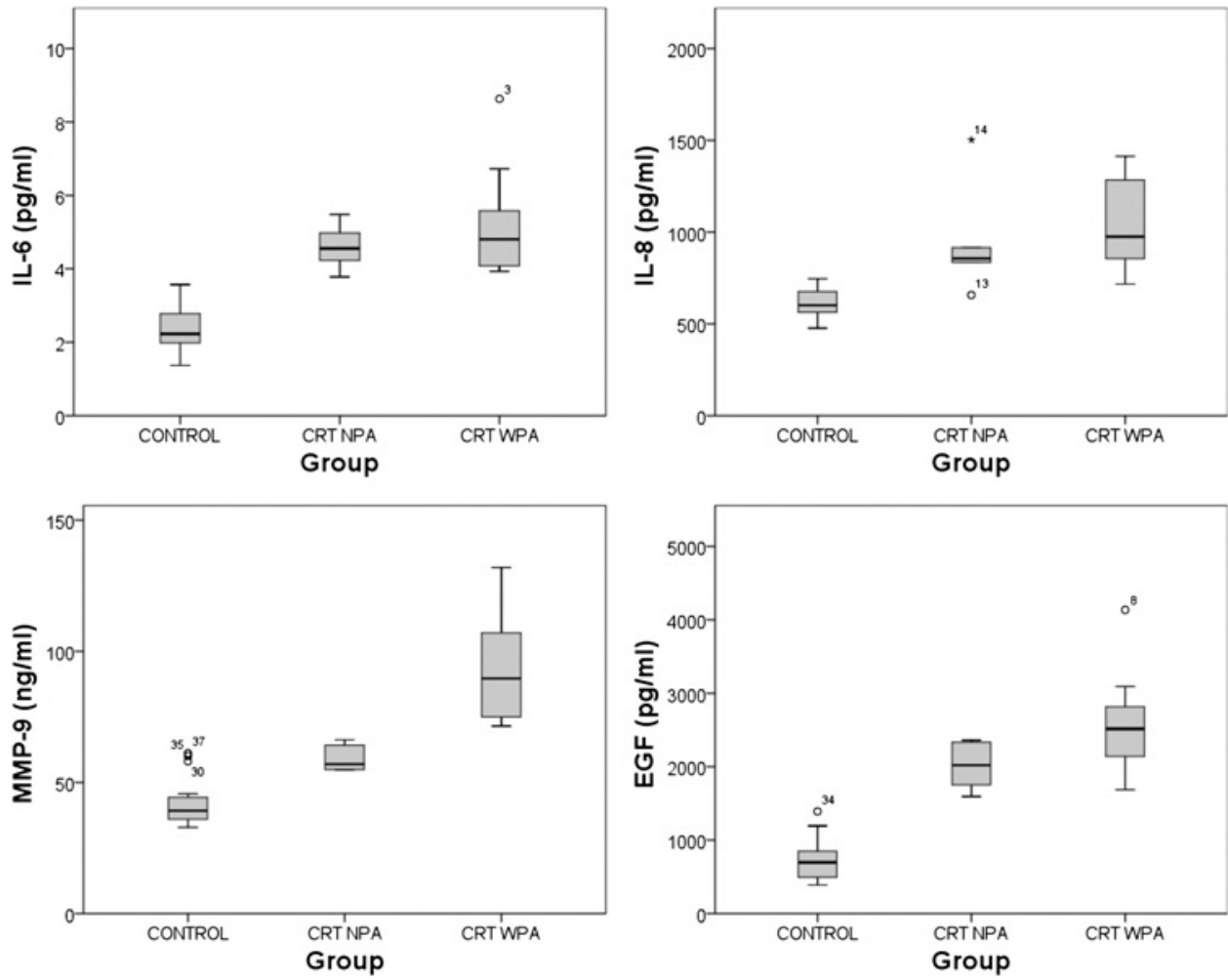

using RGP lenses. ${ }^{12}{ }^{26}$ However, we found a stronger effect, which might be explained by the reshaping epithelial effect of the reverse geometry Paragon CRT lenses and lens-induced hypoxia. In that context, IL-6 may act as a stimulating factor in epithelial cell migration, probably by a fibronectin-dependent mechanism, ${ }^{23}$ from the central cornea towards the mid-periphery during the corneal refractive therapy tissue redistribution process. $^{15} 27$

IL-8 is a potent neutrophil chemotactic and activating factor produced by many cells (including fibroblasts, endothelial cells, keratinocytes, macrophages, $\mathrm{T}$ cells, neutrophils) in response to different cytokines including IL-1 and TNF $\alpha$, or the presence of microorganisms. IL- 8 is also a constant component of the normal human tear fluid. ${ }^{23}$ In this study, the concentration of IL-8 was found to be significantly elevated by a factor of 1.6 in the samples of corneal refractive therapy patients compared with those of control subjects. Although higher, this finding is consistent with the results of previous studies using disposable and RGP contact lenses worn overnight. ${ }^{3} 22$ In our study the major elevation of this cytokine seems to occur due to a more significant mechanical effect of Paragon CRT lenses and probably lens-induced hypoxia.

MMP-9 is the primary matrix-degrading enzyme produced by the corneal epithelium and fibroblasts in response to proinflammatory stimuli such as IL- 1 and TNF $\alpha .{ }^{28}$ There is evidence to suggest that matrix metalloproteinases play a vital role in several physiological and pathological processes. They participate in extracellular matrix remodelling after wounding of the corneal surface, and MMP-9 has been found to be of central importance in catalysing the cleavage of epithelial basement membrane components. ${ }^{29}$ In the present study we have found that, for subjects wearing Paragon CRT lenses, the concentration of MMP-9 was elevated by a factor of 1.9 in a similar proportion to IL-6 cytokine. Corneal moulding and epithelial cell migration in the maintenance of the corneal refractive effect could partly explain this pattern. This increase in MMP-9 activity may facilitate the tissue redistribution induced by Paragon CRT lenses.

EGF is a mitogenic protein involved in several mechanisms such as normal cell growth, oncogenesis and wound healing. It has been shown to be a constant component of normal tears. ${ }^{30}$ EGF has been found to be upregulated in the lachrymal gland after epithelial injury ${ }^{31}$ or contact lens wear. ${ }^{3}$ In our study EGF showed the major upregulation in both contact lens groups, with an increase of $\times 2.2$ and $\times 3.4$ for the silicone-hydrogel and corneal refractive therapy subjects, respectively. These findings are consistent with the results of previous studies on the continuous wear of silicone-hydrogel lenses and overnight RGP alignment lenses, reporting an increase of $\times 2.3$ and $\times 2.4$ in EGF concentration, respectively, when compared with control subjects. ${ }^{3}$ In our study, which is the first to analyse this growth factor with orthokeratology lenses, the highest EGF levels observed in subjects wearing Paragon CRT lenses probably occur in response to the epithelial compression and the stronger mechanical effect of those lenses compared with siliconehydrogel or RGP alignment lenses. Moreover, the increased levels of EGF may have implications for the corneal epithelium redistribution process. ${ }^{27} 28$

Presumed iron deposition in the corneal epithelium was present in $60 \%$ of the cases of overnight CRT lens wear in this sample of patients. This finding is in agreement with previous literature on corneal refractive therapy and was more prominent in patients with higher baseline refractive errors. ${ }^{32}$ Consistent with other types of pigment lines found in keratoconus or refractive surgery patients, the pigmentation does not interfere with the visual axis and does not alter visual acuity in these subjects; therefore, no treatment has been necessary. ${ }^{33}$ All of the patients were informed of their condition, and none of the patients chose to discontinue their overnight corneal refractive therapy. The decreased rate of desquamation seen in typical 
contact lens wear coupled with the increased thickness of the epithelium in this area because of the Paragon CRT lens design may provide for cellular and tear stasis. This stasis most likely causes an increased rate of basal cell retention that could partly explain the increased rate of EGF found in this investigation. Moreover, given the role of MMP-9 in epithelial basal membrane management, the increased levels of MMP-9 in eyes showing iron ring pigmentation might be related to an increased rate of extracellular matrix degradation, causing iron and other deposition observed in the mid-peripheral cornea. We hypothesise that on discontinuation of contact lens wear, the cornea will return to a normal level of corneal exfoliation, and the deposition of iron will slough off with the corneal cells over time. Further studies are necessary to determine whether this will indeed occur and to determine the epidemiology and aetiology of this event in corneal refractive therapy patients.

In conclusion, this study has demonstrated increased tear levels of IL-6, IL-8, MMP-9 and EGF in the long-term period after overnight corneal refractive therapy when compared with continuous wear of silicone-hydrogel contact lenses or no lens wear. Moreover, the association of this upregulation with the level of myopia being corrected with Paragon CRT lenses reinforces the hypothesis that links this response to the physical impact of the treatment on the corneal epithelium. Although this effect might be related to the wound healing process and to increased corneal protection, a long-term effect may cause structural changes and/or may have implications for the risk of inflammation or infection. Further studies are thus required to elucidate more fully the ocular implications involved in the long-term contact lens inflammatory response.

Contributors Concept and design of the study: JG-P, MTR-A, MAP; acquisition of data: JG-P, CV-C, TSM; analysis and interpretation of data: JG-P, ILG, MTR-A, MAP; drafting the article: JG-P, MTR-A, MAP; critical revision of article: JG-P, CV-C, TSM, ILG, JMG-M, MTR-A, MAP; and final approval of article: JG-P, CV-C, TSM, ILG, JMG-M, MTR-A, MAP.

Funding This research was financed by a grant (PI-081380) from the National Institute of Health Carlos III, Madrid, Spain, Ministry of Health and Consume (General Division for Health Research Funding).

Competing interests None.

Patient consent Obtained.

Ethics approval The study was approved by the Research and Ethics Committee, University of Santiago de Compostela, Spain, and followed the tenets of the Declaration of Helsinki.

Provenance and peer review Not commissioned; externally peer reviewed.

Data sharing statement Additional data about structural changes (corneal thickness and curvature) in all groups investigated are available on request from the corresponding author: javier.gonzalez@usc.es

\section{REFERENCES}

1. Bergenske $\mathbf{P}$, Long B, Dillehay $\mathrm{S}$, et al. Long-term clinical results: 3 years of up to 30-night continuous wear of lotrafilcon A silicone hydrogel and daily wear of low-Dk/t hydrogel lenses. Eye Contact Lens 2007;33:74-80.

2. Efron N, Perez-Gomez I, Morgan PB. Confocal microscopic observations of stromal keratocytes during extended contact lens wear. Clin Exp Optom 2002;85:156-60.

3. Kallinikos P, Morgan P, Efron N. Assessment of stromal keratocytes and tear film inflammatory mediators during extended wear of contact lenses. Cornea 2006:25:1-10.

4. Efron N. Are silicone hydrogel lenses safer? Cont Lens Anterior Eye 2005;28:153-5.
5. Mountford J. An analysis of the changes in corneal shape and refractive error induced by accelerated orthokeratology. Int Contact Lens Clin 1997:24:128-43.

6. Rah MJ, Jackson JM, Jones LA, et al. Overnight orthokeratology: preliminary results of the Lenses and Overnight Orthokeratology (LOOK) study. Optom Vis Sci 2002; 79:598-605.

7. Cheung SW, Cho P. Subjective and objective assessments of the effect of orthokeratology - a cross-sectional study. Curr Eye Res 2004;28:121-7.

8. Walline JJ, Jones LA, Sinnott LT. Corneal reshaping and myopia progression. $\mathrm{Br} \mathrm{J}$ Ophthalmol 2009:93:1181-5.

9. Watt KG, Boneham GC, Swarbrick HA. Microbial keratitis in orthokeratology: the Australian experience. Clin Exp Optom 2007;90:182-7.

10. Van Meter WS, Musch DC, Jacobs DS, et al. Safety of overnight orthokeratology for myopia: a report by the American Academy of Ophthalmology. Ophthalmology 2008:115:2301-13

11. Choo JD, Holden BA, Papas EB, et al. Adhesion of Pseudomonas aeruginosa to orthokeratology and alignment lenses. Optom Vis Sci 2009;86:93-7.

12. Lema I, Durán JA, Ruiz C, et al. Inflammatory response to contact lenses in patients with keratoconus compared with myopic subjects. Cornea 2008;27:758-63.

13. Sack RA, Sathe $S$, Beaton A. Tear turnover and immune and inflammatory processes in the open-eye and closed-eye environments: relationship to extended wear contact lens use. Eye \& Contact Lens 2003;29(Suppl. 1):P80-2.

14. González-Méijome JM, Villa-Collar C, Queirós A, et al. Pilot study on the influence of corneal biomechanical properties over the short term in response to corneal refractive therapy for myopia. Cornea 2008;27:421-6.

15. Alharbi A, La Hood D, Swarbrick HA. Overnight orthokeratology lens wear can inhibit the central stromal edema response. Invest Ophthalmol Vis Sci 2005:46:2334-40.

16. Guo Y, Nguyen T, Soni S, et al. Cell shedding in overnight orthokeratology [abstract] Invest Opthalmol Vis Sci 2004;45:1581.

17. Tighe BJ. Silicone hydrogel materials - how do they work? In: Sweeney DF, ed. Silicone Hydrogels: The Rebirth of Extended Wear Contact Lenses. Oxford: Butterworth Heinemann, 2000:1-21.

18. Queirós A, González-Méijome JM, Villa-Collar C, et al. Local steepening in peripheral corneal curvature after corneal refractive therapy and LASIK. Optom Vis Sci 2010;87:432-9.

19. Lu F, Simpson T, Sorbara $L$, et al. The relationship between the treatment zone diameter and visual, optical and subjective performance in corneal refractive therapy lens wearers. Ophthalmic Physiol Opt 2007;27:568-78.

20. Tan M, Thakur A, Morris C, et al. Presence of inflammatory mediators in the tears of contact lens wearers and non-contact lens wearers. Aust NZ J Ophthalmol 1997;25 (Suppl. 1):P27-9.

21. Thakur A, Willcox MD. Contact lens wear alters the production of certain inflammatory mediators in tears. Exp Eye Res 2000;70:255-9.

22. Nieto-Bona A, González-Mesa A, Nieto-Bona MP, et al. Short-term effects of overnight orthokeratology on corneal cell morphology and corneal thickness. Cornea 2011:30:646-54

23. Nishida T, Nakamura M, Mishima $H$, et al. Interleukin 6 promotes epithelial migration by a fibronectin-dependent mechanism. J Cell Physiol 1992;153:1-5.

24. Girolamo ND, Kumar RK, Coroneo MT, et al. UVB-mediated induction of interleukin6 and -8 in pterygia and cultured human pterygium epithelial cells. Invest Ophthalmol Vis Sci 2002:43:3430-7.

25. Nakamura Y, Sotozono C, Kinoshita S. Inflammatory cytokines in normal human tears. Curr Eye Res 1998;17:673-6.

26. Schultz CL, Kunert KS. Interleukin-6 levels in tears of contact lens wearers $\mathrm{J}$ Interferon Cytokine Res 2000;20:309-10.

27. Choo JD, Caroline PJ, Harlin DD, et al. Morphologic changes in cat epithelium following continuous wear of orthokeratology lenses: a pilot study. Cont Lens Anterior Eye 2008;31:29-37.

28. Gordon GM, Ledee DR, Feuer WJ, et al. Cytokines and signaling pathways regulating matrix metalloproteinase-9 (MMP-9) expression in corneal epithelial cells. $J$ Cell Physiol 2009;221:402-11.

29. Fukuda K, Fujitsu Y, Kumagai N, et al. Inhibition of matrix metalloproteinase-3 synthesis in human conjunctival fibroblasts by interleukin-4 or interleukin-13. Invest Ophthalmol Vis Sci 2006;47:2857-64.

30. Van Setten GB, Viinikka L, Tervo T, et al. Epidermal growth factor is a constant component of normal human tear fluid. Graefes Arch Clin Exp Ophthalmol 1989:227:184-7

31. Wilson SE, Liang 0 , Kim WJ. Lacrimal gland HGF, KGF, and EGF mRNA levels increase after corneal epithelial wounding. Invest Ophthalmol Vis Sci 1999:40:2185-90.

32. Liang JB, Chou Pl, Wu R, et al. Corneal iron ring associated with orthokeratology J Cataract Refract Surg 2003;29:624-6

33. Loh A, Hadziahmetovic M, Dunaief JL. Iron homeostasis and eve disease. Biochim Biophys Acta 2009;1790:637-49. 


\title{
Tear film inflammatory mediators during continuous wear of contact lenses and corneal refractive therapy
}

Javier González-Pérez, César Villa-Collar, Tomás Sobrino Moreiras, et al.

Br J Ophthalmo/ published online June 13, 2012

doi: 10.1136/bjophthalmol-2012-301527

Updated information and services can be found at:

http://bjo.bmj.com/content/early/2012/06/12/bjophthalmol-2012-301527.full.html

These include:

References This article cites 32 articles, 5 of which can be accessed free at: http://bjo.bmj.com/content/early/2012/06/12/bjophthalmol-2012-301527.full.html\#ref-list-1

$\mathbf{P}<\mathbf{P} \quad$ Published online June 13, 2012 in advance of the print journal.

Email alerting Receive free email alerts when new articles cite this article. Sign up in service the box at the top right corner of the online article.

Topic
Collections

Articles on similar topics can be found in the following collections

\author{
Epidemiology (779 articles) \\ Optic nerve (565 articles) \\ Optics and refraction (392 articles)
}

Advance online articles have been peer reviewed, accepted for publication, edited and typeset, but have not not yet appeared in the paper journal. Advance online articles are citable and establish publication priority; they are indexed by PubMed from initial publication. Citations to Advance online articles must include the digital object identifier (DOIs) and date of initial publication.

To request permissions go to:

http://group.bmj.com/group/rights-licensing/permissions

To order reprints go to:

http://journals.bmj.com/cgi/reprintform

To subscribe to BMJ go to:

http://group.bmj.com/subscribe/ 
Notes

Advance online articles have been peer reviewed, accepted for publication, edited and typeset, but have not not yet appeared in the paper journal. Advance online articles are citable and establish publication priority; they are indexed by PubMed from initial publication. Citations to Advance online articles must include the digital object identifier (DOIs) and date of initial publication.

To request permissions go to:

http://group.bmj.com/group/rights-licensing/permissions

To order reprints go to:

http://journals.bmj.com/cgi/reprintform

To subscribe to BMJ go to:

http://group.bmj.com/subscribe/ 